\title{
The Arabic Version of the Personality Inventory for the DSM-5 (PID-5) in a Clinical Sample of United Arab Emirates (UAE) Nationals
}

Olga Coelho, MS

Rute Pires, PhD

Ana Sousa Ferreira, PhD

Bruno Gonçalves, PhD

Samia A. AlKhoori, MD

\author{
Mohamed A. Sayed, PhD \\ Amany ElRasheed, MD \\ Sara Belhoul, MS \\ Maryam AlJassmi, MS \\ Joana Stocker, PhD
}

Objectives: We aimed to test the potential of the Arabic version of the PID-5 to distinguish between clinical and non-clinical participants, as well as to examine its convergent validity and factor structure in an Emirati clinical sample. Methods: The Arabic version of the PID-5 was administered to a clinical sample comprised of 156 participants $\left(M_{\text {age }}=31.38, S D=8.99,37.8 \%\right.$ male, $62.2 \%$ female) and a community sample also comprised of 156 participants $\left(M_{\text {age }}=31.43, S D=\right.$ $9.52,37.2 \%$ male, $62.8 \%$ female). We addressed the descriptive measures, internal consistency, mean rank scores differences, convergent validity with SCL-90-R, and PID-5's factor structure. Results: As expected, the clinical sample presented statistically significantly higher scores than the non-clinical sample, with medium to high effect sizes. In addition, all the PID-5 domains showed positive correlations with most of the symptomatic constellations of the SCL-90-R as well as the PID-5 facets with all their SCL-90-R counterparts. However, our findings did not entirely replicate the PID-5 original 5-factor structure, as only a 4-factor solution was retained. Conclusions: Future studies with the Arabic PID-5 in clinical samples are needed to understand its relevance and clinical utility in Arabic countries.

Key words: DSM-5 Alternative Model of Personality Disorders; Arabic PID-5; United Arab Emirates; clinical sample Am J Health Behav. ${ }^{\mathrm{TM}}$ 2020;44(6):794-806

DOI: doi.org/10.5993/AJHB.44.6.5

$\mathrm{P}$ ersonality disorders (PDs) are among the most challenging psychiatric conditions to diagnose and treat, with patients receiving poor treatment to a life shortening condition ${ }^{1,2}$ which accounts for heavy social and economic costs., ${ }^{3,4}$ Currently considered a mental health priority, it is estimated to affect $7.8 \%$ of the general population worldwide, $545 \%$ to $51 \%$ of psychiatric outpatients in the United States, and $40 \%$ to $92 \%$ in Europe. ${ }^{6}$ In the Arabic Gulf countries, epidemiologic studies are still limited with rates on PDs in primary healthcare services ranging from 3.1\% in Saudi Ara- bia $^{7}$ to $12.7 \%$ in the United Arab Emirates, ${ }^{8}$ and $14.1 \%$ in Qatar. ${ }^{9}$ A possible explanation to these differences could be related to methodological limitations, sampling methods, and diagnostic assessments ${ }^{10}$ that lack sufficient cross-cultural validity. ${ }^{11}$ Such diagnostic inaccuracies, allied with the social stigma associated with the utilization of psychiatric and psychological services in the Middle East countries, ${ }^{12,13}$ might delay treatment interventions and negatively impact the prognosis. Complicating matters further, in multicultural countries such as the United Arab Emirates where the number of ex-

Olga Coelho, Universidade de Lisboa, Faculdade de Psicologia, CICPSI, Lisbon, Portugal. Rute Pires, Universidade de Lisboa, Faculdade de Psicologia, CICPSI, Lisbon, Portugal. Ana Sousa Ferreira, Universidade de Lisboa, Faculdade de Psicologia and Business Research Unit (BRU-IUL), Lisbon, Portugal. Bruno Gonçalves, Universidade de Lisboa, Faculdade de Psicologia, CICPSI, Lisbon, Portugal. Samia A. AlKhoori, Psychiatry Department of Rashid Hospital, Dubai, UAE. Mohamed A. Sayed, National Rehabilitation Center, Abu Dhabi, UAE. Amany ElRasheed, Al Amal Hospital, Dubai, UAE. Sara Belhoul, Al Amal Hospital, Dubai, UAE. Maryam AlJassmi, College of Natural and Health Sciences, Zayed University, Dubai, UAE. Joana Stocker, College of Natural and Health Sciences, Zayed University, Dubai, UAE.

Correspondence Ms Coelho; ocoelho@edu.ulisboa.pt 
patriates accounts for more than $80 \%$ of the country's population, ${ }^{14}$ cross-cultural differences could easily be bypassed in clinical practice based on the assumption that Western cultural frameworks are applicable to collective societies where conformity to family values overrides any individual needs. ${ }^{15}$ From this perspective, despite the efforts to establish more culturally-informed guidelines for the assessment and treatment of PDs, ${ }^{16}$ the role of the interactive dynamics between clinical manifestations along with basic biological rhythms of individuals with PDs, cultural idiosyncrasies, and relational dimensions is still to be unveiled. ${ }^{17}$

The fifth edition of the Diagnostic and Statistical Manual of Mental Disorders ${ }^{18}$ and the eleventh version of the International Classification of Mental and Behavioural Disorders ${ }^{19}$ have shown a strong commitment to capturing the complex and heterogeneous reality of PDs, shifting towards a more evidence-based dimensional paradigm that acknowledges the pivotal importance of personality traits in clinical practice. ${ }^{20}$ Throughout this transition process The Personality Inventory for the DSM-5 (PID-5) ${ }^{21}$ assumed a leading role which empirically and conceptually supported the departure from a categorical based diagnosing system of PDs to a primarily dimensional method. ${ }^{22,23}$ In fact, the PID-5 is currently the most researched measure of maladaptive personality traits and its factor structure has been confirmed across languages and nationalities (eg, for a review see Watters, Sellbom, and Bagby ${ }^{24}$ and Somma et $\mathrm{al}^{25}$ ). However, considering that personality traits can be differently promoted or suppressed across cultures, ${ }^{26}$ little is known about their relevance in non-Western clinical settings and minorities. As the authoritative measure for the assessment of criterion B of the Alternative Model for Personality Disorders (AMPD), published on DSM-5 Section III, the PID-5 is used to determine the PDs style through a hierarchical model of maladaptive personality traits. According to the AMPD the core features to determine a personality disorder diagnosis are the presence of maladaptive personality traits, along with the level of impairment on the personality function (criterion A).

The PID-5 is a self-rated inventory that characterizes 25 facets (maladaptive traits in which individuals differ) organized into 5 higher order domains of personality variation that seem to be maladaptive extremes of a normal personality's multidimensional structure, as conceptualized by the Five-Factor Model (FFM), ${ }^{27,28}$ therefore establishing an association between negative affectivity with neuroticism, detachment with extraversion, antagonism with agreeableness, disinhibition with consciousness, and psychoticism with openness. However, this last one is considered more ambiguous with some studies questioning the relation between psychoticism and openness. ${ }^{29}$ Moreover, beyond strong psychometric properties, ${ }^{30,31}$ the PID-5 also can be used as an adequate measure to capture DSM-5 Section II categorical PDs diagnosis. ${ }^{32}$

Despite its worldwide popularity, extensive empirical research, and promising results, to date only 3 studies have been published with the PID-5 in Arabic countries. After the translation of the PID5 into Arabic that was conducted in Bahrain, $\mathrm{Ku}-$ wait, and Qatar, ${ }^{33}$ a second study was conducted within the United Arab Emirates national population using the Arabic PID-5, ${ }^{34}$ and a third study using the Arabic short version of the PID- $5,{ }^{35}$ in Algeria. All these studies were conducted in community samples and, to the best of our knowledge, there are no data on clinical Arabic speaking populations. Because the aim of the PID-5 is to measure pathological personality traits, it urges the need for studies on clinical samples, for whom this measure was originally developed.

To address some of these issues, the current study's aims were to (1) test the potential of the Arabic PID-5 to distinguish between clinical and non-clinical groups, by comparing the PID-5 results on 2 matched Emirati clinical and community samples as well as (2) examine its convergent validity by correlating the PID-5 scales with the psychopathological symptomatic dimensions of the Arabic SCL-90-R ${ }^{36}$ in the clinical sample, and finally, (3) examine the PID-5 factor structure in the Emirati clinical sample.

\section{METHODS}

\section{Study Design and Participants}

The present study was limited to Emirati citizens and based on a clinical sample $(\mathrm{N}=156)$ matched with a community sample $(\mathrm{N}=156)$. The clinical sample was recruited from 3 mental health institutions in the United Arab Emirates - the Al Amal 
Psychiatric Hospital, the National Rehabilitation Center, and the psychiatric department of Rashid Hospital. Selection of the clinical participants was carried out by the institutions' psychiatrists or psychologists among the patients that, at the time of the assessment, were receiving mental health treatment, and based on clinical authority and/or clinical records. In addition, the clinicians were asked to report each patient's main diagnosis as well as any other secondary diagnosis, using the DSM5 criteria. Patients that met at least one DSM-5 mental disorder were included in the clinical sample, and those experiencing intellectual disability, schizophrenia spectrum disorder, and major and mild neurocognitive disorders were excluded from the sample. A total of 156 inpatients and outpatients were selected, aged between 18 and 61 years $\left(\mathrm{M}_{\text {age }}=31.38, \mathrm{SD}=8.99,37.8 \%\right.$ male, $62.2 \%$ female). With regards to marital status, most of the patients were single $(49.4 \%)$, that had completed high school (66.7\%), and at the time of the assessment were unemployed/housewives (43.6\%). The predominant diagnosis included substance-related and addictive disorders $(35.3 \%)$, anxiety disorders (21.8\%), and both depressive (14.7\%) and bipolar related disorders $(14.7 \%)$. The majority of the patients $(76.9 \%)$ met the criteria for at least one comorbidity, with depressive disorders (16.6\%), PD (6.6\%) and obsessive-compulsive disorders (2.5\%) being the most frequent comorbidities.

The clinical sample was subsequently matched, based on the composition of gender and age, with a community sample of 156 Emirati volunteers, aged between 18 and 57 years $\left(M_{\text {age }}=31,43, S D=9.52\right.$, $37.2 \%$ male, $62.8 \%$ female). At the time of the assessment $53.2 \%$ of the community participants were single, $57.1 \%$ had completed high school, $32.7 \%$ were employed, $22.4 \%$ were unemployed/ housewives, $42.3 \%$ were students, and $2.6 \%$ were retired/disabled. The community sample was selected from a large convenience sample of Emirati citizens $(\mathrm{N}=1090)$ recruited from Zayed University Dubai and Abu Dhabi students and their acquaintances. ${ }^{34}$ Only the participants that declared had no mental disorders were included in the community sample.

\section{Data Collection}

Patients selected by the mental health units' cli- nicians were invited to participate in the study at the end of the follow-up appointments or other consultation procedures. The nature of the study was explained, confidentiality was emphasized, and all participants signed a written consent form. Taking into consideration the time required to apply the test (approximately one hour), mutual convenient appointments were scheduled, dependent on the patients' condition and availability. Moreover, to ensure the accuracy of the responses to the test, inpatients participants were invited to take part in the study at the end of their hospitalization period, as at this stage most of the patients are free of severe psychopathological symptoms. Data collection sessions were held between May and September of 2019.

The community sample was recruited through email or in person by psychology graduate research assistants. All the community participants signed a written informed consent form, and the data collection sessions were held collectively at Zayed University Dubai and Abu Dhabi between April and September of 2019.

\section{Instruments}

Sociodemographic questionnaire. The sociodemographic questionnaire comprised questions regarding nationality, age, sex, occupational and marital status, religion, education, family, and financial situation. The participants were also asked to report if they suffered from any physical or mental disorder, and when applicable, to specify the diagnose.

Personality Inventory for DSM-5 (Al-Attiyah et
al, $^{33}$ original version of Krueger et $\left.\mathrm{al}^{21}\right)$. The PID5 is a self-report measure which operationalizes the DSM-5 model of pathological personality traits. It is comprised of 220 items, rated on a 4-point Likert scale, ranging from 0 (very false or often false) to 3 (very true or often true) that characterizes 25 empirically derived lower level facets grouped into 5 major domains of maladaptive personality variation. The instrument is to be use in adults (18 years or above) and takes 40 minutes or less to complete. The PID-5 has been studied worldwide, both in clinical and non-clinical samples, and has shown sound psychometric features such as replicable factor structure, internal consistency, convergence with personality measures, and with a broad range 
of psychopathological constructs. ${ }^{30}$ Data from the PID-5 Arabic translation study ${ }^{33}$ showed that the Cronbach's alphas of the PID-5 scales were moderate to high, ranging from .70 (manipulativeness) to .93 (attention-seeking) at the facet level, and to .92 (antagonism) to .96 (detachment) at the domain level.

Symptom Checklist-90 - Revised (Al-Behairy; 36 original version of Derrogatis ${ }^{37}$ ). The SCL-90-R is a multidimensional self-assessment questionnaire consisting of 90 items measured on a 5-point Likert scale, ranging from 0 (Never) to 4 (Extremely), assessing the presence of psychopathology and psychological distress in individuals aged 13 year and above. It comprises 9 principal symptomatic dimensions of psychopathology and 3 global indices. The dimensions are somatization, obsessions-compulsions, interpersonal sensitivity, depression, anxiety, hostility, phobic anxiety, paranoid ideation, and psychoticism. The measure also comprises an additional item scale which is a severity indicator of the individual state, although it is not related to any specific symptomatic dimension. The 3 global indices assess global distress, hardiness, and symptom free. The SCL-90-R presents good internal consistency ranging from .84 to .90 and time stability, with correlations between .80 and .90 .

\section{Data Analysis}

Statistical data analyses were performed with the IBM SPSS Statistics (v.26, SPSS Inc., Chicago, IL). To validate the PID-5 Arabic version in the Emirati population, descriptive statistics for the facets and domains were obtained and internal reliability was examined through Cronbach's alphas, in both community and clinical samples. Additionally, to test the PID-5 ability to distinguish between clinical and non-clinical samples, the mean rank score differences between the populations were calculated by the Wilcoxon Signed-Rank test, given that in both, community and clinical samples, the PID-5 scales scores in the Emirati population had shown to be highly heteroscedastic. The effect size was tested through $r=z / \sqrt{N}$, being $N$ the number of pairs without ties. PID-5 and SCL-90-R convergent validity analyses were calculated by the Spearman correlation coefficient. Finally, to examine the Arabic PID-5 factor structure we employed an exploratory factor analyses (EFA) using Equamax oblique rota- tion, and the number of factors to be selected were based on the Kaiser's, MAP, and parallel analysis criteria. The decision of how many factors to retain is crucial in EFA, ${ }^{38}$ and there are several criteria to guide the factor retention decision, not always leading to the same number of factors. EFA often is used with the correlation matrix and, in that case, one is the variance of each item/variable. Kaiser's criterion is the most used criterion, extracting only those factors with eigenvalues greater than one. Thus, Kaiser's selects factors that explain more than the variability of each item, however, this criterion tends to overestimate the number of factors. ${ }^{39}$ The minimum average partial (MAP) method $^{40}$ uses the average of squared partial correlations after each component is out. When the minimum average squared partial correlation is reached, the residual matrix resembles an identity matrix, and no further components are extracted. Another factor retention method is the parallel analysis (PA), based on the rationale that nontrivial components from real data should have larger eigenvalues than parallel components derived from random data having the same characteristics. Therefore, PA involves the construction of correlation matrices of random variables, and the average eigenvalues from the random correlation matrices are then compared to the eigenvalues from the real data correlation matrix (the first observed eigenvalue is compared to the first random eigenvalue, and so on). The factors to be retained correspond to actual eigenvalues that are greater than the parallel average random eigenvalues. Several studies have shown that MAP and PA belong to the most accurate methods set. ${ }^{38}$

\section{RESULTS}

\section{Descriptive Statistics, Internal Consistency, and Group Differences}

Table 1 presents the Arabic PID-5's scales means, SDs, and Cronbach alphas of the community and clinical samples, along with the Wilcoxon SignedRank test which allowed to compare the 2 groups differences, and respective effect size.

The facets with the higher sores were rigid perfectionism in the community sample and anxiousness in the clinical sample, while the lower scores were found on the facet callousness for both community and clinical samples. According to Wilcoxon Signed- Rank test results, the majority of the PID- 
The Arabic Version of the Personality Inventory for the DSM-5 (PID-5) in a Clinical Sample of United Arab Emirates...

Table 1

PID-5 Scales' Descriptive Statistic and Wilcoxon Signed-Rank Test

\begin{tabular}{|c|c|c|c|c|c|c|c|c|c|c|}
\hline \multirow{2}{*}{ PID-5 Facets } & \multirow{2}{*}{ Sample } & \multicolumn{3}{|c|}{ Samples measures } & \multicolumn{6}{|c|}{ Community vs Clinical } \\
\hline & & M & SD & $\alpha$ & Ranks & $\mathbf{N}$ & $\begin{array}{l}\text { Mean } \\
\text { Rank }\end{array}$ & $\mathbf{Z}$ & p & $\mathbf{r}$ \\
\hline \multirow{2}{*}{ Anhedonia } & Community & .90 & .52 & .78 & Neg. & 114 & 84.97 & \multirow{2}{*}{-7.34} & \multirow{2}{*}{.000} & \multirow{2}{*}{.60} \\
\hline & Clinic & 1.44 & .60 & .79 & Pos. & 37 & 48.36 & & & \\
\hline \multirow{2}{*}{ Anxiousness } & Community & 1.31 & .56 & .83 & Neg. & 110 & 84.18 & \multirow{2}{*}{-6.55} & \multirow{2}{*}{.000} & \multirow{2}{*}{.53} \\
\hline & Clinic & 1.83 & .65 & .86 & Pos. & 41 & 54.06 & & & \\
\hline \multirow{2}{*}{$\begin{array}{l}\text { Attention s } \\
\text { eeking }\end{array}$} & Community & 1.04 & .55 & .81 & Neg. & 100 & 80.16 & \multirow{2}{*}{-4.80} & \multirow{2}{*}{.000} & \multirow{2}{*}{.39} \\
\hline & Clinic & 1.38 & .67 & .85 & Pos. & 48 & 62.72 & & & \\
\hline \multirow{2}{*}{ Callousness } & Community & .58 & .38 & .78 & Neg. & 94 & 82.12 & \multirow{2}{*}{-4.23} & \multirow{2}{*}{.000} & \multirow{2}{*}{.35} \\
\hline & Clinic & .80 & .51 & .84 & Pos. & 54 & 61.24 & & & \\
\hline \multirow{2}{*}{$\begin{array}{l}\text { Cognitive and } \\
\text { perceptual } \\
\text { dysregulation }\end{array}$} & Community & .80 & .47 & .81 & Neg. & 101 & 86.50 & \multirow{2}{*}{-5.38} & \multirow{2}{*}{.000} & \multirow{2}{*}{.44} \\
\hline & Clinic & 1.14 & .60 & .86 & Pos. & 51 & 56.71 & & & \\
\hline \multirow{2}{*}{ Deceitfulness } & Community & .82 & .42 & .71 & Neg. & 94 & 77.36 & \multirow{2}{*}{-4.10} & \multirow{2}{*}{.000} & \\
\hline & Clinic & 1.04 & .57 & .80 & Pos. & 50 & 63.36 & & & ד \\
\hline Denressivity & Community & .63 & .45 & .86 & Neg. & 120 & 83.39 & 702 & 000 & \\
\hline Defressivity & Clinic & 1.25 & .68 & .91 & Pos. & 31 & 47.39 & -1.93 & . & .0J \\
\hline Dirtnotibilit & Community & .97 & .49 & .78 & Neg. & 121 & 83.08 & 720 & 000 & 50 \\
\hline Disti actionity & Clinic & 1.49 & .58 & .81 & Pos. & 33 & 57.05 & .50 & .000 & ק \\
\hline Focontricity & Community & .87 & .57 & .90 & Neg. & 101 & 83.68 & 504 & 000 & 11 \\
\hline 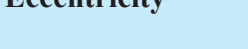 & Clinic & 1.25 & .66 & .90 & Pos. & 50 & 60.49 & -5.04 & .000 & .41 \\
\hline Emotional & Community & 1.10 & .51 & .71 & Neg. & 115 & 81.88 & 10 & 000 & 615 \\
\hline lability & Clinic & 1.66 & .66 & .79 & Pos. & 33 & 48.79 & -1.40 & . & .01 \\
\hline Grandiocity & Community & 1.17 & .54 & .70 & Neg. & 84 & 77.48 & & 005 & 10 \\
\hline 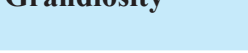 & Clinic & 1.32 & .65 & .75 & Pos. & 62 & 68.11 & -2.27 & - & .13 \\
\hline Wontilitus & Community & 1.09 & .51 & .79 & Neg. & 99 & 82.24 & 4 & 000 & 10 \\
\hline Hostminy & Clinic & 1.43 & .72 & .88 & Pos. & 50 & 60.67 & & .000 & (T⿱一⿻上丨. \\
\hline Immolcivity & Community & .94 & .51 & .72 & Neg. & 106 & 78.32 & 5 & 000 & 10 \\
\hline mptistry & Clinic & 1.38 & .67 & .77 & Pos. & 39 & 58.54 & -5.93 & .000 & .49 \\
\hline Intimacy & Community & .78 & .48 & .68 & Neg. & 87 & 84.18 & 02 & 000 & 33 \\
\hline avoidance & Clinic & 1.06 & .67 & .75 & Pos. & 58 & 56.22 & & & \\
\hline In & Community & .72 & .45 & .66 & Neg. & 114 & 81.04 & & 0 & 0 \\
\hline Irresponsidinty & Clinic & 1.20 & .56 & .66 & Pos. & 34 & 52.59 & -1.14 & .000 & .59 \\
\hline Mo & Community & 1.00 & .52 & .65 & Neg. & 77 & 77.42 & & 040 & 7 \\
\hline 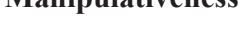 & Clinic & 1.15 & .66 & .75 & Pos. & 64 & 63.28 & 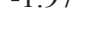 & . & .11 \\
\hline
\end{tabular}

(continued on next page) 


\begin{tabular}{|c|c|c|c|c|c|c|c|c|c|c|}
\hline \multicolumn{11}{|c|}{$\begin{array}{c}\text { Table } 1 \text { (continued) } \\
\text { PID-5 Scales' Descriptive Statistic and Wilcoxon Signed-Rank Test }\end{array}$} \\
\hline \multirow[b]{2}{*}{ PID-5 Facets } & \multirow{2}{*}{ Sample } & \multicolumn{3}{|c|}{ Samples measures } & \multicolumn{6}{|c|}{ Community vs Clinical } \\
\hline & & $\mathbf{M}$ & SD & $\alpha$ & Ranks & $\mathbf{N}$ & $\begin{array}{l}\text { Mean } \\
\text { Rank }\end{array}$ & $\mathbf{Z}$ & p & $\mathbf{r}$ \\
\hline \multirow{2}{*}{ Perseveration } & Community & 1.05 & .45 & .73 & Neg. & 101 & & \multirow{2}{*}{-4.87} & \multirow{2}{*}{.000} & \multirow{2}{*}{.40} \\
\hline & Clinic & 1.41 & .63 & .84 & Pos. & 44 & 64.24 & & & \\
\hline \multirow{2}{*}{$\begin{array}{l}\text { Restricted } \\
\text { affectivity }\end{array}$} & Community & 1.12 & .46 & .60 & Neg. & 94 & 78.50 & \multirow{2}{*}{-2.88} & \multirow{2}{*}{.004} & \multirow{2}{*}{.23} \\
\hline & Clinic & 1.29 & .57 & .65 & Pos. & 58 & 73.26 & & & \\
\hline \multirow{2}{*}{$\begin{array}{l}\text { Rigid } \\
\text { perfectionism }\end{array}$} & Community & 1.33 & .50 & .78 & Neg. & 92 & 78.22 & \multirow{2}{*}{-3.05} & \multirow{2}{*}{.002} & \multirow{2}{*}{.25} \\
\hline & Clinic & 1.53 & .65 & .86 & Pos. & 57 & 69.81 & & & \\
\hline \multirow{2}{*}{ Risk-taking } & Community & 1.14 & .43 & .76 & Neg. & 93 & 81.45 & \multirow{2}{*}{-3.77} & \multirow{2}{*}{.000} & \multirow{2}{*}{.31} \\
\hline & Clinic & 1.34 & .55 & .83 & Pos. & 56 & 64.29 & & & \\
\hline \multirow{2}{*}{$\begin{array}{l}\text { Separation } \\
\text { insecurity }\end{array}$} & Community & 1.00 & .58 & .78 & Neg. & 96 & 84.30 & \multirow{2}{*}{-5.34} & \multirow{2}{*}{.000} & \multirow{2}{*}{.44} \\
\hline & Clinic & 1.37 & .68 & .78 & Pos. & 50 & 52.77 & & & \\
\hline \multirow{2}{*}{ Submissiveness } & Community & .95 & .56 & .67 & Neg. & 103 & 77.41 & \multirow{2}{*}{-5.11} & \multirow{2}{*}{.000} & 12 \\
\hline & Clinic & 1.33 & .67 & .72 & Pos. & 43 & 64.14 & & & .42 \\
\hline Susniciousness & Community & 1.15 & .40 & .41 & Neg. & 103 & 78.17 & -506 & 000 & 42 \\
\hline Suspiciousness & Clinic & 1.42 & .50 & .52 & Pos. & 44 & 64.23 & -5.06 & .000 & .42 \\
\hline Unusual beliefs & Community & .91 & .53 & .76 & Neg. & 90 & 83.92 & -3.38 & 001 & 27 \\
\hline and experiences & Clinic & 1.15 & .68 & .82 & Pos. & 61 & 64.32 & -0.00 & .001 & .21 \\
\hline Withdrawal & Community & 1.02 & .50 & .81 & Neg. & 103 & 80.29 & -4.71 & .000 & 38 \\
\hline Withdrawal & Clinic & 1.35 & .64 & .84 & Pos. & 48 & 66.80 & $-4 . / 1$ & .000 & .38 \\
\hline Negative & Community & 1.14 & .45 & .88 & Neg. & 122 & 84.32 & -7.58 & 000 & 61 \\
\hline affectivity & Clinic & 1.62 & .55 & .90 & Pos. & 33 & 54.65 & -1.00 & & \\
\hline Detachment & Community & .90 & .39 & .86 & Neg. & 110 & 89.70 & & 000 & \\
\hline Detachment & Clinic & 1.28 & .53 & .89 & Pos. & 46 & 51.71 & -6.63 & .000 & .53 \\
\hline Antaognism & Community & 1.00 & .39 & .82 & Neg. & 90 & 87.61 & -3.12 & 000 & 25 \\
\hline Antagomsint & Clinic & 1.17 & .53 & .88 & Pos. & 66 & 66.08 & -0.12 & & \\
\hline : I & Community & .88 & .39 & .85 & Neg. & 121 & 86.63 & 11 & & \\
\hline Disinhibition & Clinic & 1.35 & .52 & .88 & Pos. & 35 & 50.40 & -7.71 & .000 & .62 \\
\hline Psuchoticism & Community & .86 & .45 & .93 & Neg. & 104 & 85.91 & 408 & 000 & 40 \\
\hline 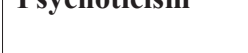 & Clinic & 1.18 & .59 & .94 & Pos. & 52 & 63.68 & -4.90 & .000 & .40 \\
\hline $\begin{array}{l}\text { Note. } \\
\text { Negative R } \\
.20<\mathrm{r} \leq .5\end{array}$ & (1) & & & & & & & & & \\
\hline
\end{tabular}

5 facets and domains mean ranks were higher in the clinical sample compared to the community sample. These comparisons were statistically significant for 20 of the 25 facets $(\mathrm{p}<.001)$ and 5 domains $(\mathrm{p}<.001)$. Regarding the effect size, we have obtained medium (.20 to .50$)$ to high (> .50) effect sizes for 23 of the 25 facets and the 5 PID5 domains. The smaller effect sizes were displayed 


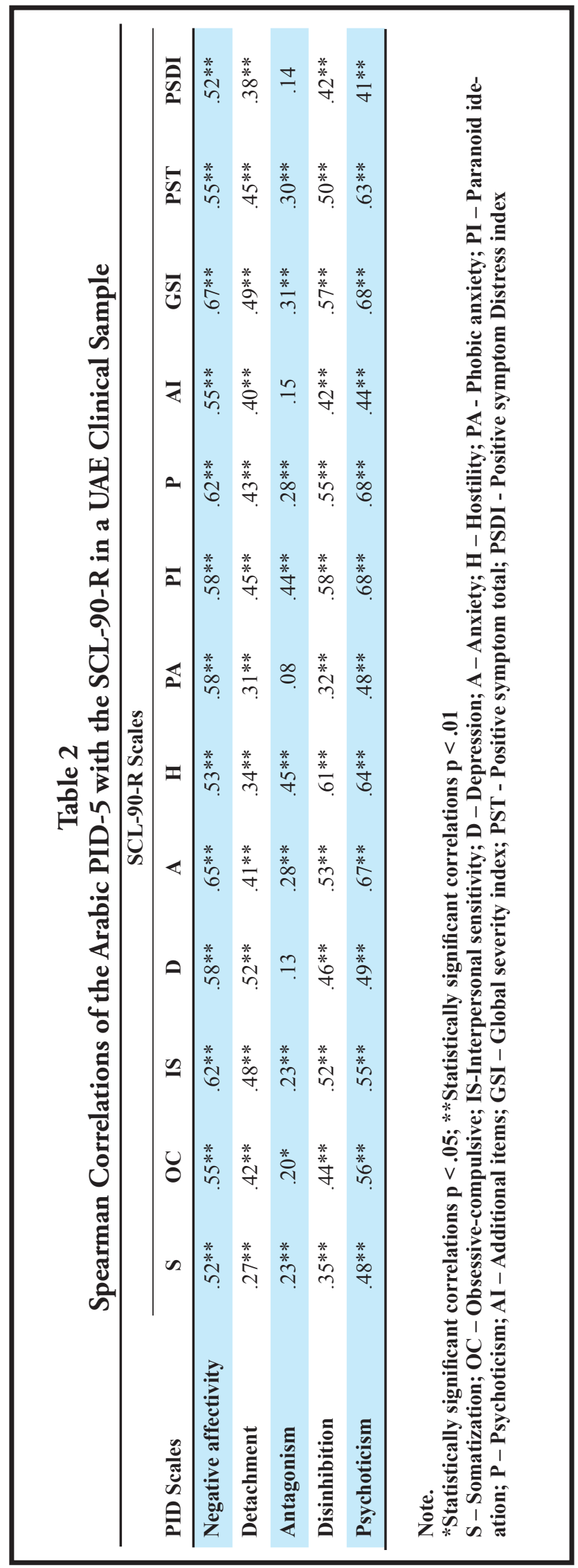

by the facets grandiosity and manipulativeness $(\leq$ .20) whereas the larger effect sizes were found on the facets depressivity, emotional lability, and anhedonia as well as on the negative affectivity and disinhibition domains.

As for the internal consistency, the alpha coefficients were acceptable to good for the majority of the PID-5 scales in the community sample ( $\geq .70$ for 19 of the facets and $\geq .80$ for the 5 domains) and in the clinical sample ( $\geq .70$ for 22 of the facets and $\geq .85$ for the 5 domains). Overall, the reliability coefficients have shown to be higher in the clinical sample than in the community sample, particularly at the trait facet level. The lowest alphas for both samples were obtained on the facets irresponsibility, restricted affectivity, and suspiciousness. These results have shown that most of the PID-5 facets and the 5 domains were reliable in both samples.

\section{Convergent Validity}

The Arabic PID-5 convergent validity was studied by correlating the PID-5 scales with the psychopathological symptomatic dimensions of the Arabic SCL-90-R in the clinical sample. At the domain level, the PID-5 negative affectivity, detachment, disinhibition, and psychoticism showed significant positive correlations with all the SCL90-R scales (Table 2) and the antagonism domain with 9 of the 13 SCL-90-R scales. The PID-5 negative affectivity presented the highest correlations with the SCL-90-R global severity index $\left(\mathrm{r}_{\mathrm{s}}=.67, \mathrm{p}\right.$ $<.01)$, anxiety $\left(\mathrm{r}_{\mathrm{s}}=.65, \mathrm{p}<.01\right)$ and interpersonal sensitivity scales $\left(\mathrm{r}_{\mathrm{s}}=.62, \mathrm{p}<.01\right)$, and detachment domain moderately and positively correlated with the SCL-90-R depression $\left(\mathrm{r}_{\mathrm{s}}=.52, \mathrm{p}<.01\right)$, global severity index $\left(\mathrm{r}_{\mathrm{s}}=.49, \mathrm{p}<.01\right)$ and interpersonal sensitivity scales $\left(\mathrm{r}_{\mathrm{s}}=.48, \mathrm{p}<.01\right)$. Also, the PID5 antagonism domain displayed moderate positive correlations with the SCL-90-R hostility $\left(\mathrm{r}_{\mathrm{s}}=.45\right.$, $\mathrm{p}<.01)$ and paranoid ideation scales $\left(\mathrm{r}_{\mathrm{s}}=.44, \mathrm{p}\right.$ $<.01$ ), whereas the PID-5 disinhibition domain presented moderate positive correlations with the SCL-90-R hostility $\left(\mathrm{r}_{\mathrm{s}}=.61, \mathrm{p}<.01\right)$, paranoid ideation $\left(\mathrm{r}_{\mathrm{s}}=.58, \mathrm{p}<.01\right)$, and global severity index scales $\left(r_{s}=.57, p<.01\right)$. Finally, the PID-5 psychoticism domain presented the highest correlations with the global severity index, paranoid ideation, and psychoticism $\left(r_{s}=.68, p<.01\right)$ SCL90-R scales. In sum, we highlight that PID-5 di- 
Table 3

Exploratory Factor Analysis with Equamax Rotation Solution of the Clinical Sample

\begin{tabular}{|c|c|c|c|c|c|}
\hline \multirow[b]{2}{*}{ PID-5 facets } & \multicolumn{4}{|c|}{ Factors } & \multirow[t]{2}{*}{ Communalities } \\
\hline & 1 & 2 & 3 & 4 & \\
\hline Anhedonia & -.01 & -.00 & .74 & .39 & .70 \\
\hline Anxiousness & -.06 & .23 & .33 & .68 & .63 \\
\hline Attention seeking & .49 & .41 & -.31 & .48 & .75 \\
\hline Callousness & .70 & .21 & .30 & -.12 & .63 \\
\hline Cognitive percep. dysreg. & .55 & .52 & .34 & .32 & .79 \\
\hline Deceitfulness & .80 & .17 & -.07 & .15 & .70 \\
\hline Depressivity & .18 & .08 & .70 & .53 & .80 \\
\hline Distractibility & .38 & .29 & .48 & .51 & .71 \\
\hline Eccentricity & .38 & .56 & .38 & .36 & .73 \\
\hline Emotional lability & .25 & .40 & .27 & .62 & .68 \\
\hline Grandiosity & .53 & .58 & -.10 & .08 & .64 \\
\hline Hostility & .62 & .32 & .40 & .19 & .68 \\
\hline Impulsivity & .66 & .11 & .30 & .25 & .60 \\
\hline Intimacy avoidance & .04 & .27 & .67 & -.10 & .53 \\
\hline Irresponsibility & .70 & -.08 & .39 & .30 & .74 \\
\hline Manipulativeness & .71 & .33 & -.21 & .04 & .65 \\
\hline Perseveration & -.07 & .72 & .27 & .31 & .70 \\
\hline Restricted affectivity & .37 & .57 & .44 & -.08 & .66 \\
\hline Rigid perfectionism & -.14 & .83 & .19 & .12 & .76 \\
\hline Risk-taking & .68 & .00 & .18 & -.23 & .55 \\
\hline Separation insecurity & .12 & .24 & -.07 & .77 & .67 \\
\hline Submissiveness & -.20 & -.11 & .05 & .70 & .54 \\
\hline Suspiciousness & .52 & .25 & .50 & .06 & .59 \\
\hline Unusual beliefs & .42 & .64 & .23 & .19 & .67 \\
\hline Withdrawal & .04 & .36 & .74 & .11 & .70 \\
\hline Eigenvalues & 9.90 & 3.10 & 2.08 & 1.70 & \\
\hline$\%$ variance explained & 39.61 & 12.39 & 8.32 & 6.80 & \\
\hline
\end{tabular}

mensions of negative affectivity, disinhibition, and psychoticism presented the strongest relations with the symptomatic constellations of the SCL-90-R as well as with the general psychopathologic indices.

With regards to the facet level, as expected, the strongest relations were found between the PID-5 and the SCL-90-R counterparts, namely the PID-5 anxiousness have shown moderate positive correlation with the SCL-90-R anxiety scale $\left(\mathrm{r}_{\mathrm{s}}=.61, \mathrm{p}<\right.$ .01 ) and the PID-5 depressivity facet displayed the highest correlation with the SCL-90-R depression scale $\left(r_{s}=.78, p<.01\right)$. Furthermore, the PID-5 hostility showed substantial correlations with its peer scale, the SCL-90-R hostility $\left(\mathrm{r}_{\mathrm{s}}=.72, \mathrm{p}<\right.$ $.01)$ as well as the PID-5 suspiciousness with the SCL-90-R paranoid ideation $\left(\mathrm{r}_{\mathrm{s}}=.68, \mathrm{p}<.01\right)$ and interpersonal sensitivity $\left(r_{s}=.61, p<.01\right)$.

Finally, perhaps due to the fact that SCL-9-R psychoticism scale captures a wide scope of symptoms ranging from light psychotic features and schizoid personality style to severe symptoms of schizophrenia spectrum disorder, all the facets of the PID-5 psychoticism domain presented moderate positive correlations with the SCL-90-R psychoticism, namely cognitive and perceptual dysregulation and eccentricity, $\left(r_{s}=.65, p<.01\right)$ along with unusual 
beliefs and experiences $\left(\mathrm{r}_{\mathrm{s}}=.53, \mathrm{p}<.01\right)$. In addition, all the PID-5 facets and domains significantly correlated with the SCL-90-R global severity index scale.

\section{Factor Structure}

The Emirati clinical sample presented a 4-factor solution suggested by the Kaiser criterion, MAP, and parallel analysis criterion (Table 3). The model displayed an excellent fit $(\mathrm{KMO}=.888)$ and the total explained variance was $(67.13 \%)$. Communalities showed that the percentage of variance explained by the extracted factors was above $50 \%$ for all the facets. Table 3 shows the 4-factor equamax rotated solution, factor loadings, eigenvalues, communalities, and the percentage of explained variance per factor in the clinical sample.

The first factor was comprised by the facets attention-seeking, callousness, cognitive and perceptual dysregulation, deceitfulness, hostility, impulsivity, irresponsibility, manipulativeness, risk-taking, and suspiciousness. Although this factor encompassed traits from the 5 PID-5 domains, it resembled a partial conjunction of the antagonism domain, if we considered that the facet grandiosity secondarily weighted (.53) on this factor, with the disinhibition domain (impulsivity, irresponsibility, and risk-taking). However, the facets cognitive and perceptual dysregulation and suspiciousness also weighed on this factor rendering its interpretation less clear.

As for the second factor onto the facets eccentricity, grandiosity, preservation, restricted affectivity, rigid perfectionism, and unusual beliefs and experiences primarily loaded, might be considered similar to the psychoticism domain, if we bear in mind that the facet cognitive and perceptual dysregulation (.52) weighted secondarily on this factor.

The third factor were the facets anhedonia, depressivity, intimacy avoidance, and withdrawal mainly loaded was akin to the detachment domain.

Finally, the facets anxiousness, distractibility, emotional lability, separation insecurity, and submissiveness all loaded onto factor 4, which resembled the negative affectivity domain, once the facet perseveration had its second main weight on this factor (.31). The facet distractibility also weighted onto factor 4 (.51), although, according to the DSM-5 model, it belongs to the disinhibition domain.
In the Emirati clinical sample, the disinhibition domain did not clearly emerge as an independent factor, with its facets weighted jointly onto factors one, 2 , and 4 . Thus, to reproduce the DSM-5 AMPD structure, the factors extraction was limited to 5-factors. However, the 5-factors obtained did not fully match the original DSM-5 trait model. For this reason, the 4-factor solution was deemed to be the most adequate and the internal consistency of its factors was calculated. The alphas obtained varied from .80 for the fourth factor (negative affectivity) to .89 for the first factor (antagonism/disinhibition), thus proving good internal reliability of the new structure in the clinical sample.

\section{DISCUSSION}

The purpose of this study was to examine the Arabic PID-5's ability to distinguish between nonclinical from clinical participants, with regards to pathological personality traits, as well as to examine its convergent validity, and factor structure cross-cultural replicability in an Emirati clinical sample.

Consistent with previous findings (eg, for a review see $\mathrm{Al}-\mathrm{Dajani}$ et $\mathrm{al}^{130}$ and Zimmermann et $\mathrm{al}^{41}$ ) the Arabic PID-5 appears to be a reliable measure of pathological personality traits in both community and clinical samples, with the internal consistency of its scales ranging from acceptable to good for the majority of the trait facets and for all the trait domains. However, the facets irresponsibility, restricted affectivity, and suspiciousness require further research as they presented the lowest alphas. In fact, several studies reported similar results in Western and non-Western samples ${ }^{11,42,43}$ perhaps due to the use of allegorical expressions such as "cold fish," "raw deal," and "skipped town" in some of their items (eg, 8, 133, 171). These expressions can be challenging to translate, especially into the Arabic language. ${ }^{44}$ Even when the meaning is preserved, the item intensity, difficulty, and standards of comparison might change across cultures and languages. ${ }^{45,46}$

As expected, the psychiatric patients sample presented statistically significant higher scores than the general community sample, with medium to high size effects in the majority of the PID-5 scales. Only the facets grandiosity and manipulativeness (facets of the antagonism domain) displayed a small effect 
size $(\leq .20)$, which could be related with situational or cultural factors, such as the tendency to respond in a socially desirable way, as social desirability tends to be higher in collectivistic cultures such as the Emirati, compared to more individualistic cultures. ${ }^{47-49}$ For example, the Arabic word "Inshallah" means "if God wills" and it is used on a daily basis to show agreement; however, it could carry a double meaning as to say "yes, if God wills," or an eloquent mean to avoid confrontation by imposing a certain sense of uncertainty towards the expected outcome. On the other hand, if we consider the high scores of anhedonia, depressivity, and emotional lability (facets associated with internalization) and the percentage of anxiety and depressive disorders in the clinical sample, perhaps the facet grandiosity has captured the vulnerable narcissism (as a lower and internalized extreme of grandiosity) of the patients sample as opposed to feelings of superiority and entitlement (as a higher and externalized extreme of grandiosity) that could, in some extent, be adaptive $e^{50,51}$ or culture-related. Overall, these results might be better explained by a continuum of common individual differences between normative and pathological personality ${ }^{52}$ grafted in a socio-cultural context that can consubstantiate their meaning. ${ }^{53}$

With regards to the convergent validity of the Arabic PID-5, the domains negative affectivity, detachment, disinhibition, and psychoticism have shown positive correlations with all the symptomatic constellations of the Arabic SCL-90-R as well as the antagonism domain with 9 of its scales. Moreover, the PID-5 facets displayed strong correlations with all their SCL-90-R counterparts, particularly with depression and hostility, in line with previous studies ${ }^{54-57}$ that confirmed important relations between pathological personality traits and mental health disorders.

These results suggest that the PID-5 has adequate criterion and convergent validity highlighting its importance in the assessment of maladaptive traits in clinical settings.

Concerning the PID-5 factor structure in the Emirati clinical sample, our findings did not reproduce a 5 -factor solution proposed by DSM-5 AMPD and replicated in most of the PID-5 studies (eg, for a review see Somma et $\mathrm{al}^{25}$ and Zimmermann et $\mathrm{al}^{41}$ ). Instead, similarly to the study conducted by Pires et al, ${ }^{58}$ we identified a 4 -factor solution that resembled the domains antagonism, psychoticism, detachment, and negative affectivity, with some facets showing a deviant loading pattern from the original structure..$^{27,59,60}$ Notably, in the first and second factors, the disinhibition domain did not clearly emerge on the 4-factor solution, with its facets loads mostly weighted on factor one, akin to the antagonism domain.

A possible explanation for this unexpected conjunction of the antagonism with the disinhibition domain, could be that individuals with narcissistic personality trait profiles, beyond showing grandiosity, callousness, and manipulativeness, can also be impulsive and behave recklessly to standout socially. Further, they can become hostile and suspicious towards the intensions and behaviours' of others. ${ }^{61}$ On the other hand, from a psychopathological point of view, this first factor seemed to group traits that characterize DSM-5 Section II Cluster B Personality Disorders, particularly the anti-social, borderline, and narcissistic personality disorders that might be related with our clinical sample composition. In this regard, Kotov et al, ${ }^{62}$ in a meta-analysis study, found high correlations between some of the 'big' personality traits with anxiety, depressive, and substance use disorders which mostly profile our clinical sample diagnosis. Furthermore, the authors stressed the lack of specificity in the personality profiles identified, and suggested that high order personality constructs are not exclusively linked to specific conditions, but they are rather meaningful under the umbrella of a more general factor of psychopathology. ${ }^{62} \mathrm{On}$ this note, several studies have pointed that Cluster B and Cluster C Personality Disorders are the most frequent neglected comorbidities among patients diagnosed with substance use disorder, anxiety, and depression in primary and secondary psychiatric care. ${ }^{28,63,64}$ As such, clinical research might consider developing combine treatment plans able to intervene on both the personality domains and the disorder that results in part from the personality itself. ${ }^{65}$

Concerning factor 2, an atypical factor loading was also obtained, which gathered traits that characterize both the schizotypal (eccentricity, unusual beliefs) and the obsessive-compulsive functioning (preservation, restricted affectivity, rigid perfec- 
tionism) similar to an imperfect combination of the compulsive and schizotypal domains initially proposed by the AMPD. ${ }^{18}$ However, as cognitive and perceptual dysregulation secondary weighted on this factor, perhaps we might consider it similar to the psychoticism domain. As noted by some studies, the psychoticism domain has been pointed as heterogeneous with deviant facet loadings ${ }^{66,67}$ and Pires et al ${ }^{58}$ reported its absence in a clinical sample. These deviations might be conceptually meaningful in Arabic countries, as some studies with the FFM in Arabic samples have failed to identify the 5 domains of personality. ${ }^{68,69}$ Therefore, given the bipolar nature of personality traits, it is not surprising that its pathological extremes, assessed by the PID5 , could also present differences in our sample, reflecting the personality complexity. ${ }^{21,27,70}$

Overall, this study indicated that there was a great deal of interaction between the domains of personality measured by the PID-5 and the psychopathology of the clinical sample. The factor solutions found in the Emirati clinical sample seemed to identify a combination of trait constellations that might be linked to the mental disorders that characterize the Emirati clinical sample, rather than to a universal structure of personality.

The present findings should be considered in the light of several limitations, as this study was a first attempt to validate the Arabic PID-5 in an Emirati clinical sample. First, the small size of the community and clinical samples. Second, the predominance of substance-related and addictive disorders (35.3\%), along with the severity of the psychiatric diagnosis and the multiple comorbidities of the inpatients, might have affected the range of PID-5 traits and symptoms. However, it is worth noting that all clinical participants were stable and about to be discharged when they completed the test. Third, although only the participants that had declared being mentally healthy were included in the community sample, no direct screening for psychopathology or previous history of utilizing mental health services has been performed.

Considering the aforementioned, our results call for future studies in Arabic speaking countries, with larger samples, and with a broader spectrum of psychiatric disorders, to clarify these unexpected results and assess the PID-5 clinical utility in Arabic mental health settings.

\section{Human Subjects Approval Statement}

All procedures were reviewed and approved by the Research Ethics Committee of Zayed University, Dubai Scientific Research Ethics Committee, and Ministry of Health and Prevention Research Ethics Committee. A written informed consent was obtained from all participants.

\section{Conflict of Interest Disclosure Statement}

The authors of this article declare no conflicts of interest.

\section{Acknowledgements}

This study was conducted in Al Amal Hospital Dubai, National Rehabilitation Centre, Rashid Hospital, and Zayed University. We thank all the staff and students that contributed to this project, especially to Amina Ahmad and Wajeeha Nasir. This study was funded by Zayed University Provost's Research Fellowship Award (R18111) to the senior author JS.

\section{References}

1. Fork ML, Hayes RD, Cang C, et al. Life expectancy at birth and all-cause mortality among people with personality disorder. J Psychosom Res. 2012;73(2):104-107.

2. Skodol AE. Personality disorders: a burden in the community, neglected in the clinic? J Clin Psychiatry. 2015;76(11):1482-1484.

3. Tyrer P, Reed GM, Crawford MJ. Classification, assessment, prevalence, and effect of personality disorders. Lancet. 2015;385:717-726.

4. Bach B, First MB. Application of the ICD-11 classification of personality disorders. BMC Psychiatry. 2018;18:351.

5. Winspei C, Bilgin A, Thompson A, et al. The prevalence of personality disorders in the community: a global systematic review and meta-analysis. Br J Psychiatry. 2020;216(2):69-78.

6. Beckwith H, Moran PF, Reilly J. Personality disorder prevalence in psychiatric outpatients: a systematic literature review. Personal Ment Health. 2014;8(2):91-101.

7. Alosaimi FD, Alzain N, Asiri S, et al. Patterns of psychiatric diagnose in inpatients and outpatients' psychometric settings in Saudi Arabia. Arch Clin Psychiatr. 2017;44(3):77-83.

8.El-Rufaie OEF, Al-Sabosy M, Abuzeid MSO, Ghubash R. Personality profile among primary care patients: experimenting with Arabic IPDE ICD-10. Acta Psychiatr Scand. 2002;105(1):37-41.

9. Bener A, Abou-Saleh MT, Dafeeah EE, Bhugra D. The prevalence and burden of psychiatric disorders in primary health care visits in Qatar: too little time? J Family Med Prim Care. 2015;4(1):89-95.

10. Volkert J, Gablonski TC, Rabung S. Prevalence of person- 
ality disorders in the general adult population in western countries: systematic review and mental analysis. $\mathrm{BrJ}$ Psychiatry. 2018;213(6):709-715.

11. Lotfi M, Bach B, Amini M, Simonsen E. Structure of DSM-5 and ICD-11 personality domains in Iranian community sample. Personal Ment Health. 2018;12(2):155169.

12. Al-Darmaki FR, Hassane S, Ahammed S, Abdullah AS. Developing counselor education identity in UAE: Evaluation of an undergraduate program. Journal for International Counselor Education. 2012;4(1):1.

13. Al-Darmaki F, Sayed MA. Counseling challenges within the cultural context of the United Arab Emirates. In Gerstein LH, Heppner PP, Ægisdóttir S, Leung MA, Norsworthy KL, eds. International Handbook of Cross-cultural Counseling: Cultural Assumptions and Practices Worldwide. Thousand Oaks, CA: Sage Publications; 2009:465474.

14. The World Bank. The World Bank Annual Report 2018. Available at: http://documents.worldbank.org/curated/ en/630671538158537244/The-World-Bank-AnnualReport-2018. Published 2018. Accessed May 12, 2020.

15. Al-Adawi S. Mental health services in Oman: the need for more cultural relevance. Oman Med J. 2017;2(32):83-85.

16. Ronningstam E, Keng SL, Ridolfi ME, et al. Cultural aspects in symptomatology, assessment and treatment of personality disorders. Cur Psychiatry Rep. 2018;20(4):22.

17. Sperandeo R, Messina G, Iennaco D, et al. What does personality mean in the context of mental health? A topic modeling approach based on abstracts published in PubMed over the last 5 years. Front Psychiatry. 2020;10:938.

18. American Psychiatric Association (APA). Diagnostic and Statistical Manual of Mental Disorders. $5^{\text {th }}$ ed. Washington, DC: American Psychiatric Publishing: 2013.

19. Word Heath Organization (WHO). International Classification of Diseases $11^{\text {th }}$ Revision (ICD-11). Geneva, Switzerland, WHO: 2018. https://icd.who.int/browse11/l-m/ en. Accessed April 20, 2020.

20. Bach B, Sellbom M, Kongerslev M, et al. Deriving ICD11 personality disorders from DSM-5 traits: initial attempt to harmonize two diagnostic systems. Acta Psychiatr Scand. 2017;136(1):108-117.

21. Krueger RF, Derringer J, Markon KE, et al. Initial construction of a maladaptive personality trait model and inventory for DSM-5. Psychol Med. 2012;42(9):18791890.

22. Skodol AE. Personality disorders in DSM-5. Annu Rev Clinic Psychol. 2012;8:317-344.

23. Watters CA, Sellbom M, Bagby RM. Comparing two domain scoring methods for the Personality Inventory for DSM-5. Psychol Assess. 2019;9(31):1125-1134.

24. Watters CA, Sellbom M, Bagby RM. A meta-analysis of the five-factor internal structure of the Personality Inventory for DSM-5. Psychol Assess. 2018;30:1255-1260.

25. Somma A, Krueger RF, Markon KE, Fossati A. The replicability of the Personality Inventory for DSM-5 domain scale factor structure in U.S. and non-U.S. samples: a quantitative review of the published literature. Psychol Assess. 2019;31(7):861-877.

26. Kitayama S, Markus HR, Matsumo H, Norasakkunkit V. Individual and collective processes in the construction of the self: self-enhancement in the United States and selfcriticism in Japan. J Pers Soc Psychol. 1997;72(6):12451267.

27. Krueger RF, Markon KE. The role of the DSM-5 personality trait model in moving toward a quantitative and empirically based approach to classifying personality and psychopathology. Annu Rev Clin Psychol. 2014;10:477501.

28. Skodol AE, Clark LA, Bender DS, et al. Proposed changes in personality and personality disorder assessment and diagnosis for DSM-5 Part I: description and rationale. Personal Disord. 2011;1(2):4-22.

29. Sleep CE, Hyatt CS, Lamkin J, et al. Examining the relations among the DSM-5 alternative model of personality, the five-factor model, and externalizing and internalizing behavior. Personal Disord. 2018; 9:379-384.

30. Al-Dajani N, Gralnick TM, Bagby M. A psychometric review of the Personality Inventory for DSM-5 (PID5): current status and future directions. J Pers Assess. 2016;98(1):62-81.

31. Zimmermann J, Mayer A, Leising D, et al. Exploring occasion specificity in the assessment of DSM-5 maladaptive personality traits. A latent state-trait analysis. Euro J Psychol Assess. 2017;33:47-54.

32. Bach B, Sellbom M, Skjernov M, Simonsen E. ICD-11 and DSM-5 personality trait domains capture categorical personality disorders: finding a common ground. Aust $N$ Z J Psychiatry. 2018;52(5):425-434.

33. Al-Attiyah AA, Megreya AM, Alrashidi M, et al. The psychometric properties of an Arabic version of the Personality Inventory for DSM-5 (PID-5) across three Arabic-speaking Middle Eastern countries. Int J Cult Ment Health. 2017;10(2):197-205.

34. Coelho O, Pires R, Sousa Ferreira A, et al. The Arabic version of The Personality Inventory for the DSM-5 (PID-5) in a community sample of United Arab Emirates Nationals. Clin Pract Epidemiol Ment Health. 2020;16(1):180188.

35. Bach B, El-Abiddine, FZ. Empirical structure of DSM-5 and ICD-11 personality disorder traits in Arabic-speaking Algerian culture, Int J Ment Health. 2020;49(2):186-200.

36. Al-Behairy AA. Symptom Checklist-9O Revised (SCL-90). Cairo, Egypt: Maktabat Al-Nahda Al-Misriya: 1984.

37. Derrogatis LR. Symptom Checklist 90-R: Administration, Scoring and Procedures Manual I for the Revised Version of the SCL-90. Baltimore, MD: John Hopkins University Press: 1977.

38. Hayton JC, Allen DG, Scarpello V. Factor retention decisions in exploratory factor analysis: a tutorial on parallel analysis. Organ Res Methods. 2004;7(2):191-205.

39. Horn JL. A rationale and test for the number of factors in factor analysis. Psychometrika. 1965;32:179-185.

40. Velicer WF. Determining the number of components from the matrix of partial correlations. Psychometrika. 1976;41:321-327.

41. Zimmermann J, Kerber A, Rek K, et al. A brief but comprehensive review of research on The Alternative DSM5 Model for Personality Disorders. Curr Psychiatry Rep. 2019;21(9):21-92.

42. Roskam I, Galdiolo S, Hansenne M, et al. The psychometric properties of the French version of the Personality Inventory for DSM-5. PLoS One. 2015;10(7):e0133413. 
The Arabic Version of the Personality Inventory for the DSM-5 (PID-5) in a Clinical Sample of United Arab Emirates...

43. Rowiński T, Kowalska-Dąbrowska M, Strus WS, et al. Measurement of pathological personality traits according to Section III of the DSM-5: a Polish adaptation of PID5. Part II - empirical results. Psychiatr Pol. 2019;53(1):2348.

44. Ghazala H. Allegory in Arabic expressions of speech and silence. https://translationjournal.net/journal/20arabic. htm. Published April 2002. Accessed February 15, 2020.

45. McCrae RR. Exploring trait assessment of samples, persons, and cultures. J Pers Assess. 2013;95(6):556-570.

46. Van de Vijver FJR, Leung K. Methods and data analysis of comparative research. In Berry JW, Poortinga YH, Pandey J, eds. Handbook of Cross-cultural Psychology: Theory and Method. Boston, MA: Allyn \& Bacon; 1997:257300.

47. Bernardi R. Associations between Hofstede's cultural constructs and social desirability response bias. J Bus Ethics. 2006;65:43-53.

48. Mahmood SI, Daim AS, Borleffs JCC, et al. The transferability of Western concepts to other cultures: validation of the Zuckerman-Kuhlman Personality Questionnaire in a Saudi Arabic context. Med Teach. 2015;37(1):61-74.

49. Van Hemert DA, Van de Vijver FJR, Poortinga YH, Georgas, J. Structural and functional equivalence of the Eysenck Personality Questionnaire within and between countries. Pers and Individ Dif. 2002;33:1229-1249.

50. Miller JD, Lynam DR, Campbell WK. Measures of narcissism and their relations to DSM-5 pathological traits: a critical reappraisal. Assessment. 2016;23(1):3-9.

51. Witt EA, Hopwood CJ, Morey LC, et al. Psychometric characteristics and clinical correlates of NEO-PI-R fearless dominance and impulsive anti-sociality in the collaborative longitudinal personality disorders study. Psychol Assess. 2010;22(3):559-568.

52. Oltmanns JR, Smith GT, Oltmanns TF, Widiger TA. General factors of psychopathology, personality, and personality disorder: across domain comparisons. Clin Psychol Sci. 2018;6(4):581-589.

53. La Roche MJ, Fuentes M, Hinton D. A cultural examination of the DSM-5: research and clinical implications for cultural minorities. Prof Psychol Res Pr. 2015;3(48):183189.

54. Dunne AL, Gilbert F, Daffern M. Investigating the relationship between DSM-5 Personality Disorder domains and facets and aggression in an offender population using the Personality Inventory for the DSM-5. J Pers Disord. 2018;32:668-693.

55. Fowler JC, Madan A, Allen JG, et al. Differentiating bipolar disorder from borderline personality disorder: diagnostic accuracy of the difficulty in emotion regulation scale and personality inventory for DSM-5. J Affect Disord. 2019;245:856-860.

56. Seyed HSG, Merghati KE, Hosseinnezhad S, et al. Per- sonality traits and substance use disorders: comparative study with drug user and non-drug user population. Pers Individ Dif. 2019;148:50-56.

57. Skjernov M, Bach B, Fink P, et al. DSM-5 Personality disorders and traits in patients with severe health anxiety. J Nerv Ment Dis. 2020;208(2):108-117.

58. Pires R, Sousa Ferreira A, Gonçalves B, et al. The Portuguese version of the Personality Inventory for DSM-5 in a community and a clinical sample. Personal Ment Health. 2019;13(1):40-52.

59. Hopwood CH, Donnellan MB. How should the internal structure of personality inventories be evaluated? Pers Soc Psychol Rev. 2010;14(3):332-46.

60. Turkheimer E, Ford D, Oltmanns TF. Regional analysis of self-reported personality disorder. J Pers. 2008;76(6):1587-1622.

61. Hopwood CJ, Schade N, Krueger RF, et al. Connecting DSM-5 personality traits and pathological beliefs: toward a unifying model. J Psychopathol Behav Assess. 2013;35(2):162-172.

62. Kotov R, Gamez W, Schmidt F, Watson D. Linking 'big' personality traits to anxiety, depressive, and substance use disorders: a meta-analysis. Psychol Bull. 2010;136:768821.

63. Asp M, Lindqvist D, Fernström J, et al. Recognition of personality disorder and anxiety disorder comorbidity in patients treated for depression in secondary psychiatric care. PLoS One. 2020;15(1):e0227364.

64. De Carlo V, Calati R, Serreti A. Socio-demographic and clinical predictors of non-response/non-remission in treatment resistant depressed patients: a systematic review. Psychiatry Res. 2016;240:421-430.

65. Widiger TA, Sellbom M, Chmielewski M, et al. Personality in a Hierarchical Model of Psychopathology. Clinical Psychological Science. 2019;7(1):77-92.

66. Adhiatma W, Pinaima R, Siregal LB, et al. Exploratory factor analysis for Indonesian version of PID-5. Indonesian Psychological Journal. 2014;29(4):199-206.

67. Bach B, Sellbom M, Simonsen E. Personality Inventory for DSM-5 (PID-5) in clinical versus nonclinical individuals: generalizability of psychometric features. Assessment. 2019;25(3):815-825.

68. Alansari BM. The psychometric Properties of NEO FiveFactor Inventory (NEO-FFI-S) based on the Kuwaiti Society. Psychological Quarterly. 1997;7:277-310.

69. Latzman RD, Megreya AM, Hecht LK, et al. Self-reported psychopathy in the middle east: A cross-national comparison across Egypt, Saudi Arabia, and the United States. BMC Psychology. 2015;3:37.

70. Gutierrez F, Aluja A, Peri JM, et al. Psychometric properties of the Spanish PID-5 in a clinical and a community sample. Assessment. 2017;24(3):326-336. 